\title{
CYCLOSPORINE TREATMENT IMPROVES CARDIAC FUNCTION AND ATTENUATES MITOCHONDRIAL INJURY IN ASPHYXIATED NEWBORN PIGLETS DURING REOXYGENATION
}

\author{
R.S. Gill ${ }^{1}$, N. Manouchehri ${ }^{1}$, T.-F. Lee ${ }^{2}$, J.-Q. Liu ${ }^{2}$, W.J. Cho ${ }^{3}$, D. Bigam ${ }^{1}$, P.-Y. Cheung ${ }^{1,2}$ \\ ${ }^{I}$ Surgery, ${ }^{2}$ Pediatrics, ${ }^{3}$ Cell Biology, University of Alberta, Edmonton, AB, Canada
}

Background: Asphyxiated neonates often have myocardial depression which is a significant cause of morbidity and mortality. Cardioprotective effects of cyclosporine have been observed in adult patients and animals with myocardial infarction. However, the cardioprotective effect of cyclosporine in neonates has not yet been studied.

Objectives: We hypothesize that cyclosporine will improve cardiac function and reduce myocardial injury in asphyxiated newborn piglets.

Methods: Thirty-six piglets (1-4 days-old) were acutely instrumented for continuous monitoring of cardiac output and systemic arterial pressure. After stabilization, normocapnic alveolar hypoxia (10-15\% oxygen) was instituted for $2 \mathrm{~h}$ followed by reoxygenation with $100 \%(0.5 \mathrm{~h})$, then $21 \%(3.5 \mathrm{~h})$ oxygen. Piglets were block-randomized to receive intravenous boluses of cyclosporine A $(2.5,10$ or $25 \mathrm{mg} / \mathrm{kg})$ or normal saline (control) at $5 \mathrm{~min}$ of reoxygenation ( $\mathrm{n}=8 /$ group). Cardiomyocyte mitochondria were assessed for morphology and markers of injury.

Results: Hypoxic piglets had cardiogenic shock (cardiac output $40-48 \%$ of baseline), hypotension (mean arterial pressure $30 \mathrm{mmHg}$ ) and acidosis $(\mathrm{pH}$ 7.04). Cyclosporine-treatment caused bell-shaped improvements in cardiac output and systemic oxygen delivery ( $p<0.05$ vs. controls). Although histological features of myocardial injury were not different among groups, severe damage was observed in mitochondria of control piglets but attenuated in that of cyclosporine $(10 \mathrm{mg} / \mathrm{kg})$ treatment. Markers of mitochondrial injury including cytosol cytochrome-c and lipid hydroperoxides were significantly decreased and mitochondrial aconitase activity was preserved in cyclosporine-treated piglets compared to controls (all $\mathrm{P}<$ $0.05)$.

Conclusions: Post-resuscitation administration of cyclosporine causes preservation of cardiac function and attenuates cardiac mitochondrial injury in newborn piglets following asphyxia-reoxygenation. 